\title{
Research Paper: Reliability of Semmes-Weinstein Monofilaments and Tuning Fork on Pressure and Vibration Sensation Measurements in Diabetic Patients
}

\author{
Masumeh Bagherzadeh Cham ${ }^{1,2}$ (D), Mohammad Ali Mohseni-Bandpei ${ }^{3,4^{*}}$ (D), Mahmood Bahramizadeh ${ }^{5}$ (D), Saeed Kalbasi ${ }^{6}$ (D), Akbar Biglarian ${ }^{7}$ (C) \\ 1. Neuromusculoskeletal Research Center, Iran University of Medical Sciences, Tehran, Iran. \\ 2. Department of Orthotics \& Prosthetics, School of Rehabilitation Sciences, Iran University of Medical Sciences, Tehran, Iran. \\ 3. Pediatric Neurorehabilitation Research Center, University of Social Welfare and Rehabilitation Sciences, Tehran, Iran. \\ 4. University Institute of Physical Therapy, Faculty of Allied Health Sciences, University of Lahore, Lahore, Pakistan. \\ 5. Department of Orthotics and Prosthetics, University of Social Welfare and Rehabilitation Sciences, Tehran, Iran. \\ 6. Research Institute for Endocrine Sciences, Shahid Beheshti University of Medical Sciences, Tehran, Iran. \\ 7. Department of Biostatistics, Social Determinants of Health Research Center, University of Social Welfare and Rehabilitation Sciences, Tehran, Iran.
}

\begin{tabular}{|c|c|}
\hline $\begin{array}{l}\text { Use your device to scan } \\
\text { and read the article online }\end{array}$ & Citation Bagherzadeh Cham M, Mohseni-Bandpei MA, Bahramizadeh M, Kalbasi S, Biglarian A. Reliability of Semmes- \\
\hline$\square+7 \square$ & Weinstein Monofilaments and Tuning Fork on Pressure and Vibration Sensation Measurements in Diabetic Patients. Iranian \\
\hline 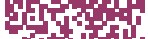 & Rehabilitation Journal. 2019; 17(1):1-8. http://dx.doi.org/10.32598/irj.17.1.1 \\
\hline 臬4 & deli http://dx.doi.org/10.32598/irj.17.1.1 \\
\hline
\end{tabular}

\section{(i) (3)}

Article info:

Received: 20 Oct 2018

Accepted: 23 Jan 2019

Available Online: 01 Mar 2019

\section{Keywords:}

Pressure sensation, Vibration sensation, Observer variations, Diabetes Mellitus

\section{A B STRACT}

Objectives: Sensory neuropathy is the major cause of ulceration in diabetic patients. Periodical sensory examination is an appropriate method to detect neuropathy and decrease the risk of diabetic foot ulcer. Semmes-Weinstein Monofilaments (SWM) and tuning fork on/off test are widely used to assess pressure and vibration sensitivity. The present study evaluated the interand intra-rater reliability of SWM and tuning fork to measure pressure and vibration sensations in diabetic patients.

Methods: Following ethical approval, 34 diabetic patients without foot ulceration were recruited. Feet kit SWM and tuning fork were used for pressure and vibration sensation evaluation, respectively. Measurements were performed twice on the first session with an hour interval to assess within-day reliability and the third one was demonstrated 7 days later to assess between-days reliability.

Results: Within-day Intra-class Correlation Coefficient (ICC) analysis indicated excellent levels of inter- and intra- rater reliability (ICC $>0.75$ ) for feet kit SWM in all foot regions and $128 \mathrm{~Hz}$ and $256 \mathrm{~Hz}$ tuning forks. Excellent between-days intra-rater reliability (ICC $>0.75)$ was also obtained for SWM; however, the ICC of tuning fork was moderate. Between-days interrater reliability of SWM and tuning fork were high and poor, respectively.

Discussion: The obtained results indicated that SWM and tuning fork seemed to be highly reliable to measure pressure and vibration sensations in diabetic patients. However, further studies are required to support the results of current study.

* Corresponding Author: 


\section{Highlights}

- Semmes-Weinstein monofilaments are reliable to measure pressure sensation in diabetic patients.

- The tuning fork is reliable to measure vibration sensations in diabetic patients.

\section{Plain Language Summary}

Sensory neuropathy is one of the main causes of foot ulcer in diabetic patients. Thus, diabetic patients should be regularly evaluated for sensory perception. One of the simplest ways of sensory evaluation is to examine the pressure sensation by monofilaments and vibration sensation by tuning fork. These two tools (monofilaments and tuning fork) should have sufficient accuracy and reliability to evaluate sensory perception in diabetic patients. In this study, the reliability of these two tools was evaluated for sensory evaluation of diabetic patients and the results showed that they have sufficient reliability in several sessions (intervals of about 7 days). Also, these tests were performed by several experts that showed no significant differences in the obtained results.

\section{Introduction}

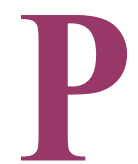

eripheral Sensory Neuropathy (PSN) is the main risk factor for foot ulceration and lower extremity amputations in diabetic patients [1]. Peripheral neuropathy involves sensory, motor and autonomous systems and causes decreased pressure sensation, increased vibration perception threshold, foot deformities, and dry skin [1].

Previous research recognized sensory neuropathy as the major cause of ulceration in diabetic patients [1, 2]. Approximately $50 \%$ of diabetic patients experience peripheral neuropathy and more than $50 \%$ of them experience symptomatic neuropathy [3]. Early diagnosis of PSN is important in diabetic patients to prevent diabetic foot problems. Periodical sensory examinations are appropriate to detect neuropathy and decrease the risk of diabetic foot ulcer. According to the Polish Diabetes Society, diabetic patient's feet should be examined at every medical visit [4].

Nerve conduction velocity is a gold standard test to detect neuropathy [5]. This test is a complex, costly, and time consuming method and not suitable for clinical evaluations [6]. The ideal instrument for neuropathy examination should be easily accessible, simple to use, and reliable with high sensitivity and specificity. Several instruments are frequently used to screen peripheral neuropathies such as monofilaments, tuning fork, and biothesiometry $[3,6]$.

Previous studies demonstrated that Semmes-Weinstein Monofilament (SWM) and tuning fork are appropriate tools for the clinical evaluation of pressure and vibration sense, respectively [1, 3, 5-8]. Monofilaments and tuning fork are non-invasive, available and easy to use instruments [5, 6, 9]. Monofilaments, often called SWM, are calibrated, single fiber nylon threads, identified by values ranging from 1.65 to 6.65 that generate reproducible buckling stress. The higher the value of the monofilament, the stiffer and more difficult it is to bend [5]. Monofilaments are used to detect the pressure sensation in neuropathic patients [10]. Reliability of SWM in healthy and elderly people was evaluated by the previous studies [4, 11-13].

The graduated tuning fork and tuning fork on/off tests are available in every dialectology clinic. Tuning fork test determines the ability of subjects to discriminate vibration sensation. Limited studies evaluated the reliability of graduated tuning fork for the detection of fracture- and immune-mediated polyneuropathies; however, the tuning fork on/off test reliability was overlooked [14, 15]. Previous studies demonstrated that SWM and tuning fork are appropriate tools to clinically evaluate pressure and vibration senses, respectively [1,3-7]. Reliability of SWM and tuning fork on/off tests are important in the evaluation of periodic clinical sensation. The present study aimed to determine the within- and between-subject intra- and interrater reliability of pressure and vibration senses measurements with SWM presented with a 6-piece foot kit and 128 and $256 \mathrm{~Hz}$ tuning fork in diabetic patients.

\section{Methods}

\section{Study participants}

Thirty-four patients with type 2 diabetes, aged 40-60 years were recruited from Taban diabetic center, Tehran City, Iran. Participants with skin ulcer or scar in the test- 
ing site, vascular diseases (absence of at least one foot pulse) and other causes of neuropathy such as alcoholism, thyroid disease, lumbar or cervical radiculopathy, inflammatory and infection diseases were excluded [16]. Neuropathy severity was assessed by the Toronto Clinical Neuropathy Score (TCNS) to maintain the patient's condition during the two test sessions.

TCNS consists of 13 items; 6 items focusing on symptoms, two items testing reflexes and 5 items assessing sensation. Symptom and sensation items answered as yes or no are given scores 0 and 1 , respectively. Normal reflex item is scored 0 , reduced reflex item is scored 1 and the lack of reflex item is scored 2. The maximum achievable score is 13 [17]. Participants' demographic data were recorded at the first session. The study was approved by the Medical Ethics Committee of the University of Social Welfare and Rehabilitation Sciences, Tehran, Iran. All subjects provided their informed consent and signed the consent form.

\section{Equipment}

Six feet kit SWM (North Coast Medical Inc.; USA) consisting of sizes $2.83,3.61,4.17,4.56,5.07$, and 6.10 was used to evaluate the pressure sensation of foot plantar surface [18]. Four regions of both feet including heel, medial and lateral of forefoot and hallux were examined by SWM [18]. Moreover, $128 \mathrm{~Hz}$ and $256 \mathrm{~Hz}$ tuning fork (Surgicon; Germany) were employed to evaluate vibration sensation in the hallux region of both feet $[9,19]$.

\section{Study procedure}

Each participant was examined twice on the same day with an hour interval by one examiner in respect of inter- and intra-rater reliability. The third measurement was demonstrated 7 days later to assess between-days reliability $[4,18]$. First and second examination sessions were conducted in the same room and under the same condition. The testing protocol was described briefly for all participants and a simple test was performed on the patient's hand to become familiarize with monofilament and tuning fork test in the first session.

The patients were requested to remove shoes and socks, lay on the bed and close eyes; then, they were instructed to say "yes" each time they felt the pressure of monofilament. Both examiners randomly selected SWM size and foot region for pressure sensation test. Examiners placed the monofilament on the plantar surface of the foot and pressed it to bend into a C-shape for approximately 11/2 seconds. Pressure sensation tests were repeated three times at each foot region with each monofilament [20, 21]. After an hour interval, the second measurement was performed using SWM and tuning fork.

Vibration testing by $128 \mathrm{~Hz}$ and $256 \mathrm{~Hz}$ tuning fork on/ off method was applied to the hallux of both feet. The patient was requested to report the lack/existence of vibration [5]. Vibration sensation test was repeated three times for both tuning fork frequencies, while vibration was randomly off in one test. To evaluate the betweendays reliability of kit SWM for feet and tuning fork, a similar protocol was performed with a 7 days interval.

\section{Statistical analysis}

Descriptive statistic were used to describe the demographic data. Intra-class Correlation Coefficients (ICC) and Standard Error of the Mean (SEM) were calculated to evaluate within- and between-subject inter- and intrarater reliability of SWM and tuning fork test. Based on Rosner's classification, ICC $\geq 0.75$ represent excellent, $0.4 \leq \mathrm{ICC}<0.75$ indicate fair to good, and $<0.4$ demonstrate poor reliability [22]. Statistical analyses were performed using SPSS.

\section{Results}

Thirty-four patients (11 men and 23 female) were included in the study. The study participants' demographic data are listed in Table 1. Neuropathy severity was similar in both sessions among all study participants based on TCNS $(\mathrm{P}>0.05)$. The ICC analysis results suggested excellent levels of inter- and intra-rater reliability (ICC $\geq 0.75$ ) with respect to SWM in all regions of feet and both tuning fork frequencies (Tables 2 and 3).

Between-days intra-rater reliability of SWM was excellent, except for right medial forefoot and left lateral forefoot (ICC $=0.73$ ) (Table 2). Between-days intra-rater ICC of both tuning fork frequencies were moderate $(0.4 \leq \mathrm{ICC}<0.75)$ (Table 3$)$. Between-days inter-rater reliability of SWM was high, except for medial forefoot of both feet (ICC $=0.62$ and 0.46 , respectively) (Table 2 ), and it was poor by tuning fork (Table 3 ).

Within-day intra-rater ICC values of pressure sensation with all sizes of SWM were 0.93 (right heel, medial forefoot and hallux) to 0.89 (left lateral of forefoot); withinday inter-rater reliability were 0.92 (right hallux) to 0.79 (right Heel). Between-days intra-rater reliability range were 0.91 (left heel) to 0.73 (right lateral forefoot and left medial forefoot) and inter-rater ICC were 0.84 (left heel) to 0.46 (left medial forefoot). 
Table 1. Participants' demographic characteristics

\begin{tabular}{cccc}
\hline Variable & Mean \pm SD & Minimum & Maximum \\
\hline Age, $y$ & $51.64 \pm 6.52$ & 40 & 60 \\
Weight, $\mathrm{kg}$ & $72.67 \pm 8.85$ & 56 & 93 \\
Height, $\mathrm{cm}$ & $166.55 \pm 7.64$ & 155 & 180 \\
\hline Body mass index, $\mathrm{kg} / \mathrm{m}^{2}$ & $26.22 \pm 2.98$ & 21.46 & 32.37 \\
\hline Disease duration, $\mathrm{y}$ & $11.70 \pm 5.80$ & 3 & 30 \\
\hline
\end{tabular}

Iranian Rehabilitation Journal

Within-day intra-rater reliability range of vibration sensation with both tuning fork frequency was $0.92(256 \mathrm{~Hz}$ in right hallux) to $0.82(128 \mathrm{~Hz}$ in right hallux), and interrater ICC was $0.92(256 \mathrm{~Hz}$ in left hallux) to 0.76 (128 $\mathrm{Hz}$ in right hallux). Between-days intra-rater reliability range was 0.71 (128 Hz in right hallux) to $0.46(128 \mathrm{~Hz}$ in left hallux) and inter-rater ICCs were $0.47(256 \mathrm{~Hz}$ in left hallux) to 0.18 (128 Hz right hallux).

\section{Discussion}

High levels of within- and between-days inter-rater reliability were detected for the SWM in the present study. Our result was in line with other studies that reported high inter-rater reliability [11, 12, 18, 23]. However, it was in contrast with Rozental et al. and Collins et al. studies that documented low inter-rater reliability $[13,24]$.

Examiner professionalism was important in measuring pressure sensation by SWM and affected the level of data reliability $[12,25]$. Robert et al. study demonstrated that a professionally trained examiner positively affected inter-rater reliability. Examiners of the current study (M.B, A.M) were expert to evaluate pressure and vibration sensation by SWM and tuning fork and were trained by a professional neurologist [25].

Table 2. ICC and SEM for inter-and intra-rater reliability of measuring pressure sensation with Semmes-Weinstein monofilaments

\begin{tabular}{|c|c|c|c|c|c|c|c|c|c|c|c|c|c|c|c|c|c|}
\hline \multirow{3}{*}{ 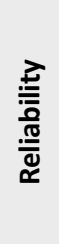 } & \multirow{3}{*}{ đ্ّ } & \multicolumn{8}{|c|}{ Right Foot } & \multicolumn{8}{|c|}{ Left Foot } \\
\hline & & \multicolumn{2}{|c|}{ Heel } & \multicolumn{2}{|c|}{$\begin{array}{l}\text { Medial } \\
\text { Forefoot }\end{array}$} & \multicolumn{2}{|c|}{$\begin{array}{l}\text { Lateral } \\
\text { Forefoot }\end{array}$} & \multicolumn{2}{|c|}{ Hallux } & \multicolumn{2}{|c|}{ Heel } & \multicolumn{2}{|c|}{$\begin{array}{l}\text { Medial } \\
\text { Forefoot }\end{array}$} & \multicolumn{2}{|c|}{$\begin{array}{l}\text { Lateral } \\
\text { Forefoot }\end{array}$} & \multicolumn{2}{|c|}{ Hallux } \\
\hline & & ICC & SEM & ICC & SEM & ICC & SEM & ICC & SEM & ICC & SEM & ICC & SEM & ICC & SEM & ICC & SEM \\
\hline
\end{tabular}
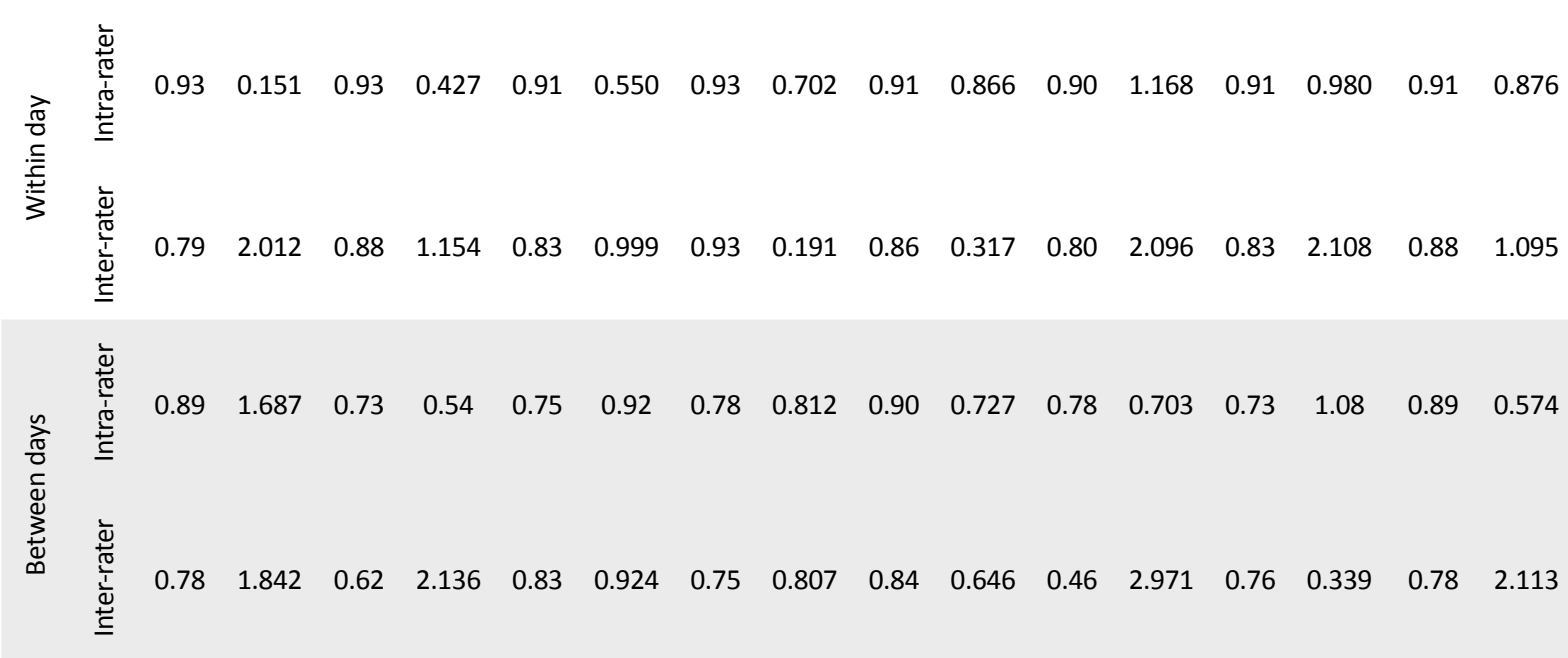
Table 3. ICC and SEM for within-and between-days inter-and intra-rater reliability of measuring vibration sensation with both tuning fork frequencies

\begin{tabular}{|c|c|c|c|c|c|c|c|c|c|}
\hline \multirow{3}{*}{ Reliability } & \multirow{3}{*}{ Frequency } & \multicolumn{4}{|c|}{ Right Foot } & \multicolumn{4}{|c|}{ Left Foot } \\
\hline & & \multicolumn{2}{|c|}{$128 \mathrm{~Hz}$} & \multicolumn{2}{|c|}{$256 \mathrm{~Hz}$} & \multicolumn{2}{|c|}{$128 \mathrm{~Hz}$} & \multicolumn{2}{|c|}{$256 \mathrm{~Hz}$} \\
\hline & & ICC & SEM & ICC & SEM & ICC & SEM & ICC & SEM \\
\hline \multirow{2}{*}{ Within day } & Intra-rater & 0.82 & 0.587 & 0.91 & 0.623 & 0.91 & 0.587 & 0.90 & 0.657 \\
\hline & Inter-rater & 0.76 & 1.697 & 0.86 & 1.296 & 0.83 & 2.286 & 0.90 & 0.784 \\
\hline \multirow{2}{*}{$\begin{array}{c}\text { Between } \\
\text { days }\end{array}$} & Intra-rater & 0.71 & 2.986 & 0.46 & 2.546 & 0.52 & 5.763 & 0.66 & 2.021 \\
\hline & Inter-rater & 0.44 & 3.743 & 0.18 & 2.512 & 0.42 & 5.279 & 0.47 & 1.193 \\
\hline
\end{tabular}

Iranian Rehabilitation Journal

The participant's individual variation affected the reliability [26]. All studies that reported low inter-rater reliability of SWM have examined healthy people [13, 24]. Individual variation was low in healthy people; however, other studies that examined the reliability of SWM in elderly [11] or patients $[12,18,23]$ reported high levels of inter-rater reliability. The present study evaluated the diabetic patients with and without neuropathy and the ICC revealed a high inter-rater reliability.

A number of monofilament, test location and examiner may affect the level of reliability. A prolonged period of the test may lead to fatigue and decrees participant's concentration. Although long-time participant's immobilization for testing decreased lower limb blood circulation and foot temperature. Decreased skin temperature is affected by plantar sensation [27].

Furthermore, increased number of tests and examiners enhanced participant's learning. Anderson et al. [12] and Roberts et al. [25] performed test-retest assessment by 5 and 9 expert and trained examiners, respectively. These studies reported high inter-rater reliability; however, Collins et al. [13] evaluated inter-rater reliability by two examiners and reported a low reliability. In the present study, 6 sizes of SWM were tested in the 4 areas of both feet by the first examiner and retested after 5 minutes by the second examiner.

A maximum time lasted approximately 5 minutes for all tests performed by each examiner. Moreover, temperature, room condition and participant's position were similar in all tests.

Test location may affect the reliability score [13]; Collins et al. indicated stronger reliability for some sub-tests [13]. Heel, metatarsal head and the plantar surface of toes (especially hallux toes) are evaluated for testing plantar sensation in diabetic patients [28]. Previous studies argued that heel, hallux and metatarsal head were reliable testing regions [18]. In the current study, within- and between-days inter-rater reliability were excellent in all areas except for medial forefoot of both feet in betweendays inter-rater reliability that was moderate.

The intra-rater reliability demonstrated high ICC in within- and between-days examination, similar to previous studies [13, 18, 23]. Only the reliability of right medial forefoot and left lateral forefoot were moderate. Collins et al. assessed the between-days intra-rater reliability in healthy people and reported good reliability for the right foot and poor-to-moderate reliability for the left foot. Individual's variation and examiner proficient affected the reliability $[25,26]$. Studies demonstrated that vibration sensation test is appropriate for neuropathy diagnosis in diabetic patients [28]. Reliability of graduate tuning fork was assessed in diabetic and polyneuropathy patients [14].

There is no data available on the reliability of on/off method of tuning fork for vibration sensation evaluation in diabetic patients. Results of the present study demonstrated that within-day intra-and inter-rater reliability of tuning fork were excellent. However, between-days intra- and inter-rater reliability were moderate and poor, respectively.

An analysis of 3 cohort studies indicated that disagreement in tuning fork result was associated with age, height and the area of vibration test. In addition, the vibration sensation test by tuning fork results depended on examiner experience and were highly subjective [28]. In our study, both evaluators were expert and only the vibration sensation of hallux wax examined. Percentage of the correct answer on total on/off test of each tuning fork was calculated and the personal opinion of the examiner did not affect the results. 


\section{Conclusion}

The current study was demonstrated to investigate reliability of within- and between-days inter- and intra-rater reliability in diabetic patients by SWM and tuning fork test. The between-days inter- and intra-rater reliability of the tuning fork were low. The sensation of diabetic patients must be periodically evaluated to understand whether SWM is a reliable tool for this reason.

\section{Ethical Considerations}

\section{Compliance with ethical guidelines}

All procedures performed in studies involving human participants were in accordance with the ethical standards of the University of Social Welfare and Rehabilitation Sciences research committee and with the 2013 Helsinki declaration. Informed consent was obtained from all patients included in the study.

\section{Funding}

This research did not receive any specific grant from funding agencies in the public, commercial, or not-forprofit sectors.

\section{Authors contributions}

Designing and performing tests: Masumeh Bagherzadeh Cham and Akbar Biglarian; Collecting the data and co-writting the paper: Masumeh Bagherzadeh Cham and Saeed Kalbasi; Performing the analysis: Akbar Biglarian; and Supervising the research: Mohammad Ali Mohseni-Bandpei and Mahmood Bahramizadeh.

\section{Conflict of interest}

The authors declare no conflict of interest.

\section{Acknowledgements}

The authors would like to thank the Iran National Science Foundation (INSF), Tehran, Iran for supporting this study.

\section{References:}

[1] Frykberg RG, Zgonis T, Armstrong DG, Driver VR, Giurini JM, Kravitz SR, et al. Diabetic foot disorders: A clinical practice guideline. Journal of Foot and Ankle Surgery. 2006; 45(5):S1-66. [DOI:10.1016/S1067-2516(07)60001-5]
[2] Frykberg RG, Lavery LA, Pham H, Harvey C, Harkless L, Veves A. Role of neuropathy and high foot pressures in diabetic foot ulceration. Diabetes Care. 1998; 21(10):1714-9. [DOI:10.2337/diacare.21.10.1714] [PMID]

[3] Llewelyn JG. The diabetic neuropathies: Types, diagnosis and management. Journal of Neurology, Neurosurgery, and Psychiatry. 2003; 74(S2):ii15-9. [PMID] [PMCID]

[4] Tracey E, Greene A, Doty R. Optimizing reliability and sensitivity of semmes-weinstein monofilaments for establishing point tactile thresholds. Physiology and Behavior. 2012; 105(4):982-6. [DOI:10.1016/j.physbeh.2011.11.002] [PMID]

[5] Perkins B, Olaleye D, Zinman B, Bril V. Simple screening tests for peripheral neuropathy in the diabetes clinic. Diabetes Care. 2001; 24(2):250-6. [DOI:10.2337/diacare.24.2.250] [PMID]

[6] Dros J, Wewerinke A, Bindels P, van Weert H. Accuracy of monofilament testing to diagnose peripheral neuropathy: A systematic review. Annals of Family Medicine. 2009; 7(1):5558. [DOI:10.1370/afm.1016] [PMID] [PMCID]

[7] Temlett JA. An assessment of vibration threshold using a biothesiometer compared to a $\mathrm{C} 128-\mathrm{Hz}$ tuning fork. Journal of Clinical Neuroscience. 2009; 16(11):1435-8. [DOI:10.1016/j. jocn.2009.03.010] [PMID]

[8] Boike AM, Hall JO. A practical guide for examining and treating the diabetic foot. Cleveland Clinic Journal of Medicine. 2002; 69(4):342-8. [DOI:10.3949/ccjm.69.4.342]

[9] Meijer JW, Smit AJ, Lefrandt JD, Van Der Hoeven JH, Hoogenberg K, Links TP. Back to basics in diagnosing diabetic polyneuropathy with the tuning fork! Diabetes Care. 2005; 28(9):2201-5. [DOI:10.2337/diacare.28.9.2201] [PMID]

[10] Abbott CA, Carrington AL, Ashe H, Bath S, Every LC, Griffiths J, et al. The north-west diabetes foot care study: Incidence of, and risk factors for, new diabetic foot ulceration in a community-based patient cohort. Diabetic Medicine. 2002 19(5):377-84. [DOI:10.1046/j.1464-5491.2002.00698.x] [PMID]

[11] Shaffer S, Harrison A, Brown K, Brennan K. Reliability and validity of semmes-weinstein monofilament testing in older community-dwelling adults. Journal of Geriatric Physical Therapy. 2005; 28(3):112-3. [DOI:10.1519/00139143200512000-00019]

[12] Anderson A, Croft R. Reliability of semmes weinstein monofilament and ballpoint sensory testing, and voluntary muscle testing in Bangladesh. Leprosy Review. 1999; 70(3):305-13. [DOI:10.5935/0305-7518.19990034] [PMID]

[13] Collins S, Visscher P, De Vet HC, Zuurmond WW, Perez RS. Reliability of the semmes weinstein monofilaments to measure coetaneous sensibility in the feet of healthy subjects. Disability and Rehabilitation. 2010; 32(24):2019-27. [DOI:10.3109/09638281003797406] [PMID]

[14] Merkies IS, Schmitz PI, van der Meche FG, van Doorn PA. Reliability and responsiveness of a graduated tuning fork in immune mediated polyneuropathies. The Inflammatory Neuropathy Cause and Treatment (INCAT) Group. Journal of Neurology, Neurosurgery, and Psychiatry. 2000; 68(5):66971. [DOI:10.1136/jnnp.68.5.669] [PMID] [PMCID]

[15] Van Den Berg CD. The reliability of the tuning fork and therapeutic ultrasound to determine the most reliable meth- 
ods of fracture diagnosis [PhD theis]. University of Johannesburg; 2009 .

[16] Kamei N, Yamane K, Nakanishi S, Yamashita Y, Tamura T, Ohshita K, et al. Effectiveness of semmes-weinstein monofilament examination for diabetic peripheral neuropathy screening. Journal of Diabetes and its Complications. 2005; 19(1):4753. [DOI:10.1016/j.jdiacomp.2003.12.006] [PMID]

[17] Bril V, Perkins BA. Validation of the Toronto clinical scoring system for diabetic polyneuropathy. Diabetes Care. 2002; 25(11):2048-52. [DOI:10.2337/diacare.25.11.2048] [PMID]

[18] Mawdsley RH, Behm-Pugh AT, Campbell JD, Carroll CR, Chernikovich KA, Mowbray MK, et al. Reliability of measurements with semmes-weinstein monofilaments in individuals with diabetes. Physical and Occupational Therapy in Geriatrics. 2004; 22(3):19-36. [DOI:10.1080/J148v22n03_02]

[19] Armstrong DG, Hussain SK, Middleton J, Peters EJ, Wunderlich RP, Lavery LA. Vibration perception threshold: Are multiple sites of testing superior to single site testing on diabetic foot examination? Ostomy Wound Management. 1998; 44(5):70-4. [PMID]

[20] Miranda-Palma B, Sosenko JM, Bowker JH, Mizel MS, Boulton AJ. A comparison of the monofilament with other testing modalities for foot ulcer susceptibility. Diabetes Research and Clinical Practice. 2005; 70(1):8-12. [DOI:10.1016/j. diabres.2005.02.013] [PMID]

[21] Lee S, Kim H, Choi S, Park Y, Kim Y, Cho B. Clinical usefulness of the two-site Semmes-Weinstein monofilament test for detecting diabetic peripheral neuropathy. Journal of Korean Medical Science. 2003; 18(1):103-7. [DOI:10.3346/ jkms.2003.18.1.103] [PMID] [PMCID]

[22] Rosner B. The intraclass correlation coefficient. In: Taylor M, editor. Fundamentals of Biostatistics. Cambridge: Harvard University; 2010.

[23] van Brakel WH, Khawas IB, Gurung KS, Kets CM, van Leerdam ME, Drever W. Intra- and inter-tester reliability of sensibility testing in leprosy. International Journal of Leprosy and Other Mycobacterial Diseases. 1996; 64(3):287-98.

[24] Rozental TD, Beredjiklian PK, Guyette TM, Weiland AJ Intra- and interobserver reliability of sensibility testing in asymptomatic individuals. Annals of Plastic Surgery. 2000; 44(6):605-9. [DOI:10.1097/00000637-200044060-00005] [PMID]

[25] Roberts A, Nicholls P, Maddali P, Van Brakel W. Ensuring inter-tester reliability of voluntary muscle and monofilament sensory testing in the INFIR Cohort study. Leprosy Review. 2007; 78(2):122-30. [PMID]

[26] de Vet HC, Terwee CB, Knol DL, Bouter LM. When to use agreement versus reliability measures. Journal of Clinical Epidemiology. 2006; 59(10):1033-9. [DOI:10.1016/j.jclinepi.2005.10.015] [PMID]

[27] Doeland HJ, Nauta JJ, van Zandbergen JB, van der Eerden HA, van Diemen NG, Bertelsmann FW, et al. The relationship of cold and warmth cutaneous sensation to age and gender. Muscle \& Nerve. 1989; 12(9):712-5. [DOI:10.1002/ mus.880120903] [PMID]

[28] Cornblath D. Diabetic neuropathy: Diagnostic methods Advanced Studies in Medicine. 2004; 4(8A):650-61. 
This Page Intentionally Left Blank 